\title{
Ciclo de mudas e taxas de crescimento de Metamysidopsis elongata atlantica (Crustacea, Mysidacea) cultivado em diferentes temperaturas e salinidades
}

\author{
Andréa Mara da Silva Gama ${ }^{1}$, Mônica Adelina Montú ${ }^{1,3}$ \& Fernando D’Incao²
}

1. Fundação Universidade Federal do Rio Grande (FURG), Depto de Oceanografia, Lab. de Zooplâncton, Caixa Postal 474, 96201-900 Rio Grande, RS, Brasil. (andreagama@terra.com.br)

2. Fundação Universidade Federal do Rio Grande (FURG), Depto de Oceanografia, Lab. de Crustáceos Decápodes, Caixa Postal 474, 96201-900 Rio Grande, RS, Brasil. (docdinca@super.furg.br)

3. In memoriam.

\begin{abstract}
Moulting cycle and growth rate of Metamysidopsis elongata atlantica (Crustacea, Mysidacea) reared at different temperatures and salinities. Metamysidopsis elongata atlantica (Bascescu, 1968) was reared in the laboratory at different combinations of temperature $\left(13,20\right.$, and $\left.25^{\circ} \mathrm{C}\right)$ and salinity $(25,30$, and $35 \%$ ) to estimate the intermoult period and carapace growth rate. Temperature affected both intermoult period and growth rate of the animals. Mysids reared at $13^{\circ} \mathrm{C}$ had longer intermoult periods than those reared at 20 and $25^{\circ} \mathrm{C}$. While animals reared at $13^{\circ} \mathrm{C}$ had the longest longevity, females did not develop marsupium when reared at this temperature. Growth rate estimated for mysids aged between 0 to 14 days (age at the onset of maturity) was $0.02 \mathrm{~mm} / \mathrm{day}, 0.04 \mathrm{~mm} /$ day, and $0.05 \mathrm{~mm} /$ day for animals reared at $13^{\circ} \mathrm{C}, 20^{\circ} \mathrm{C}$, and $25^{\circ} \mathrm{C}$, respectively.
\end{abstract}

KEYWORDS. Crustacea, Mysidae, Metamysidopsis elongata atlantica, growth rate, molts.

RESUMO. Metamysidopsis elongata atlantica (Bascescu, 1968) foi cultivada em laboratório em diferentes combinações de temperatura $\left(13,20\right.$, and $\left.25^{\circ} \mathrm{C}\right)$ e salinidade $(25,30$, and $35 \%$ ) para estimar o período de intermuda e a taxa de crescimento da carapaça. A temperatura afetou o período de intermuda e a taxa de crescimento dos animais. Misidiáceos criados a $13^{\circ} \mathrm{C}$ tiveram maiores períodos de intermuda que aqueles cultivados a 20 and $25^{\circ} \mathrm{C}$. Enquanto animais criados a $13^{\circ} \mathrm{C}$ tiveram maior longevidade, fêmeas não desenvolvem marsúpio quando cultivadas nesta temperatura. A taxa de crescimento estimada para misidáceos entre 0 a 14 dias (idade no início da maturidade) foi 0,02 $\mathrm{mm} / \mathrm{dia}, 0,04 \mathrm{~mm} / \mathrm{dia}$, e $0,05 \mathrm{~mm} /$ dia para animais cultivados respectivamente a $13^{\circ} \mathrm{C}, 20^{\circ} \mathrm{C}$ e $25^{\circ} \mathrm{C}$.

PALAVRAS-CHAVE. Crustacea, Mysidae, Metamysidopsis elongata atlantica, taxa de crescimento, mudas.

Populações de Metamysidopsis elongata atlantica (Bacescu, 1968) foram registradas na praia do Cassino, Rio Grande do Sul, Brasil por Gama \& ZamBoni (1999), habitando a zona de arrebentação sob a forma de densas manchas. Estes organismos possuem grande potencial para o uso em testes de toxicidade, por apresentarem extrema sensibilidade a baixas concentrações de substâncias tóxicas (NIMMO \& HAMAKER, 1982), motivo pelo qual GAMA \& ZAMBONI (1999) desenvolveram um protocolo para seu cultivo em laboratório.

Misidáceos carregam seus ovos em um marsúpio, sendo os juvenis liberados pelas fêmeas com forma semelhante ao adulto. Por possuírem exoesqueleto, o crescimento de misidáceos é descrito pelas suas mudas (Clutter \& Theilacker, 1971). O crescimento de alguns crustáceos (MAUChline, 1976, 1977) pode ser descrito em termos de períodos de intermudas e fatores de crescimento. Período de intermuda é definido como tempo decorrente entre mudas sucessivas, é freqüente e ocorre em intervalos regulares até a maturação sexual, variando entre 12 e 14 dias para M. elongata atlantica (GAMA \& ZAmBOni, 1999). Após a maturação sexual, as mudas tornam-se assíncronas, coincidindo com o período da desova das fêmeas (Lussier et al., 1988). O fator de crescimento é a porcentagem de aumento de tamanho em comprimento do animal entre cada muda. Para Neomysis integer (Leach, 1814), AstTHORSSON \& RALPH (1984) observaram que a duração do período de intermuda depende diretamente do comprimento do organismo e da temperatura em que eles crescem. Organismos que vivem em águas quentes atingem a maturidade sexual mais cedo, apresentando menos mudas até a maturidade. GAMA et al. (2001) registraram, com salinidade de 30\% e temperatura de $20^{\circ} \mathrm{C}$, cinco mudas em M. elongata atlantica até a maturação sexual (14 dias).

O conhecimento do ciclo de mudas torna-se importante para poder-se avaliar os efeitos da variação do ambiente na duração dos diferentes estágios. O método de estudo de crescimento através do uso das exúvias torna-se uma alternativa para a pesquisa do crescimento de crustáceos em cultivos de laboratório, sem a necessidade do manuseio do indivíduo.

O objetivo é fornecer informações sobre o ciclo de mudas e taxa média de crescimento da carapaça em $M$. elongata atlantica cultivado em laboratório a temperaturas de 13,20 e $25 \pm 1^{\circ} \mathrm{C}$ e salinidades de 25,30 e $35 \%$.

\section{MATERIAL E MÉTODOS}

Fêmeas de Metamysidopsis elongata atlantica com marsúpio esbranquiçado foram removidos dos tanques de cultivo e isoladas até a eclosão dos juvenis. Os recém-eclodidos foram retirados aleatoriamente e separados em recipientes de polipropileno contendo água 
do mar filtrada, sendo alimentados diariamente com náuplios de Artemia sp., seguindo o protocolo de GAMA $\&$ ZAMBONI (1999). Foram cultivados em salinidades de 25,30 e $35 \%$, sendo a primeira obtida por diluição e a última por salinização. Para obter as diferentes temperaturas, foram usadas três salas com aclimatização controlada de 13,20 e $25 \pm 1^{\circ} \mathrm{C}$.

Foram individualizados 30 espécimes para cada combinação de salinidade e temperatura. Diariamente as exúvias e os organismos mortos foram retirados, registrados e acondicionados em tubos de Eppendorf contendo formol a 4\% tamponado com borato de sódio, para medições posteriores. As medidas, efetuadas com ocular micrométrica em microscópio estereoscópico, foram tomadas da extremidade distal do rostro até o extremo posterior da carapaça.

Nair (1939) e ClutTer \& TheILACKer (1971) citaram mudas ocorrendo dentro do marsúpio de misidáceos antes da expulsão dos juvenis. Neste trabalho computou-se apenas as mudas ocorridas após a eclosão dos juvenis, não considerando as mudas intramarsupiais. O cálculo da taxa média de crescimento da carapaça foi realizado do nascimento do indivíduo até a maturação (14 dias), com Lt$\mathrm{Li} / \mathrm{t}$; onde $\mathrm{Lt}=$ comprimento final de carapaça; $\mathrm{Li}=$ comprimento inicial de carapaça e $\mathrm{t}=$ número de dias. Os pré-requisitos de normalidade e homocedasticidade foram testados e aceitos pelas rotinas de Kolmogorov-Smirnov $(p<0,2)$ e Levene $(p>0,05)$. UmaANOVA-multifatorial com nível de significância de 5\%, seguida de um teste de Tukey (95\%) (STAT SOFT, 2001) foram usados para verificar o efeito da salinidade e temperatura no incremento médio do comprimento de carapaça (CC) dos indivíduos.

\section{RESULTADOS}

A análise de variância mostrou que existe pelo menos um tratamento estatisticamente diferente $(\mathrm{p}=0,000)$, sendo então aplicado o teste a posteriori de Tukey para detectar as diferenças estatísticas entre os tratamentos. Foram identificados três grupos significativamente diferentes, sendo (temperatura e salinidade): grupo $1\left(13^{\circ} \mathrm{C}\right.$ e $25 \%$; $13^{\circ} \mathrm{C}$ e $30 \%$ e $13^{\circ} \mathrm{C}$ e $35 \%$ o); grupo $2\left(20^{\circ} \mathrm{C}\right.$ e $25 \%$; $20^{\circ} \mathrm{C}$ e $30 \%$ e $20^{\circ} \mathrm{C}$ e $35 \%$ ) e grupo $3\left(25^{\circ} \mathrm{C}\right.$ e $25 \% ; 25^{\circ} \mathrm{C}$ e $30 \%$ e $25^{\circ} \mathrm{C}$ e $35 \%$ ). Observa-se que dentro do grupo 1 os tratamentos 1, 2 e 3 são estatisticamente iguais. Da mesma forma, os tratamentos 4, 5 e 6 , que formam o grupo 2 e os tratamentos 7, 8 e 9, pertencentes ao grupo 3, são também estatisticamente iguais (Tab. II). Pode se observar que a temperatura influi significativamente no incremento da muda e que a temperatura de $25 \pm 1^{\circ} \mathrm{C}$ mostrou maior influência no crescimento, não importando a salinidade.

Os misidáceos cultivados em temperaturas de $13 \pm$ $1^{\circ} \mathrm{C}$ mostraram tendências de períodos de intermudas relativamente maiores quando comparados com as temperaturas de 20 e $25 \pm 1{ }^{\circ} \mathrm{C}$ (Tab. I). Ocorreram três mudas até o período da diferenciação sexual para 20 e 25 $\pm 1^{\circ} \mathrm{C}$, sendo que os misidáceos cultivados a $13^{\circ} \mathrm{C}$ não apresentaram desenvolvimento do marsúpio (característica diagnóstica de maturação sexual para fêmeas) até o final do experimento. Na temperatura de $13^{\circ} \mathrm{C}$ e salinidade de $25 \%$ ocorreram 10 mudas; para salinidade de $30 \%$ apenas 8 mudas, e a $35 \%$ o número de mudas caiu para 6 . No grupo de misidáceos mantidos em temperaturas mais elevadas $\left(25 \pm 1^{\circ} \mathrm{C}\right)$, os organismos mudaram com maior freqüência (um período de 2,4 dias

Tabela I. Média e desvio padrão (dp) do número de mudas (dias) de Metamysidopsis elongata atlantica cultivado em laboratório com temperatura $\left(\mathrm{T}^{\circ} \mathrm{C}\right)$ de 13,20 e $25 \pm 1^{\circ} \mathrm{C}$ e salinidade $(\mathrm{Sal})$ de 25,30 e $35 \%$.

\begin{tabular}{|c|c|c|c|c|c|c|c|c|c|c|c|c|c|c|c|c|c|c|}
\hline & $\mathrm{T}^{\circ} \mathrm{C}$ & Sal & $\mathrm{T}^{\circ} \mathrm{C}$ & Sal & $\mathrm{T}^{\circ} \mathrm{C}$ & Sal & $\mathrm{T}^{\circ} \mathrm{C}$ & Sal & $\mathrm{T}^{\circ} \mathrm{C}$ & Sal & $\mathrm{T}^{\circ} \mathrm{C}$ & Sal & $\mathrm{T}^{\circ} \mathrm{C}$ & Sal & $\mathrm{T}^{\circ} \mathrm{C}$ & Sal & $\mathrm{T}^{\circ} \mathrm{C}$ & Sal \\
\hline & 13 & 25 & 13 & 30 & 13 & 35 & 20 & 25 & 20 & 30 & 20 & 35 & 25 & 25 & 25 & 30 & 25 & 35 \\
\hline mudas & média & $\mathrm{dp}$ & média & $\mathrm{dp}$ & média & $\mathrm{dp}$ & média & $\mathrm{dp}$ & média & $\mathrm{dp}$ & média & $\mathrm{dp}$ & média & $\mathrm{dp}$ & média & $\mathrm{dp}$ & média & $\mathrm{dp}$ \\
\hline 1 & 6,33 & 2,52 & 5,69 & 2,81 & 5,30 & 2,22 & 2,93 & 2,17 & 3,00 & 0,00 & 2,37 & 1,11 & 2,07 & 0,36 & 2,53 & 0,88 & 2,20 & 0,60 \\
\hline 2 & 12,53 & 3,61 & 10,72 & 3,08 & 10,07 & 1,96 & 5,67 & 2,17 & 6,00 & 0,23 & 5,57 & 1,17 & 4,47 & 0,96 & 4,67 & 1,16 & 4,37 & 0,87 \\
\hline 3 & 18,89 & 5,04 & 16,82 & 3,52 & 14,54 & 2,90 & 8,69 & 1,84 & 8,23 & 0,45 & 8,64 & 1,37 & 6,93 & 1,00 & 6,97 & 1,56 & 6,63 & 1,20 \\
\hline 4 & 27,20 & 9,48 & 24,48 & 6,49 & 18,70 & 3,03 & 11,00 & 1,96 & 10,89 & 1,01 & 11,56 & 1,75 & 9,67 & 1,62 & 9,72 & 2,12 & 9,31 & 2,14 \\
\hline 5 & 39,40 & 12,03 & 33,25 & 6,28 & 26,13 & 5,21 & 13,73 & 2,28 & 14,82 & 1,23 & 14,59 & 2,10 & 12,33 & 1,94 & 13,21 & 2,22 & 12,28 & 2,36 \\
\hline 6 & 49,89 & 11,08 & 45,75 & 5,72 & 37,33 & 10,26 & 16,77 & 2,44 & 18,95 & 2,27 & 17,88 & 2,25 & 15,62 & 2,45 & 16,04 & 2,41 & 15,52 & 2,15 \\
\hline 7 & 62,33 & 10,48 & 49,00 & 0,00 & & & 20,17 & 2,41 & 25,47 & 2,26 & 21,58 & 2,55 & 19,14 & 3,12 & 19,08 & 2,76 & 18,96 & 1,72 \\
\hline 8 & 70,00 & 11,37 & 59,00 & 0,00 & & & 24,05 & 2,73 & 31,24 & 3,55 & 25,65 & 2,63 & 22,56 & 3,80 & 23,87 & 4,57 & 22,76 & 2,65 \\
\hline 9 & 71,00 & 3,74 & & & & & 28,65 & 3,07 & 36,78 & 4,08 & 29,82 & 3,23 & 25,86 & 3,16 & 27,76 & 5,49 & 26,71 & 2,84 \\
\hline 10 & 80,00 & 0,00 & & & & & 33,12 & 3,68 & 42,98 & 4,51 & 34,90 & 4,06 & 28,71 & 2,81 & 31,47 & 5,96 & 31,53 & 3,65 \\
\hline 11 & & & & & & & 37,47 & 4,10 & 47,62 & 4,05 & 41,64 & 5,26 & 31,25 & 1,64 & 34,43 & 2,47 & 36,42 & 4,73 \\
\hline 12 & & & & & & & 42,65 & 4,04 & 51,81 & 2,88 & 46,25 & 5,79 & & & 38,00 & 2,98 & 41,29 & 6,71 \\
\hline 13 & & & & & & & 47,93 & 4,49 & & & 50,38 & 5,24 & & & 42,78 & 3,91 & 46,67 & 6,16 \\
\hline 14 & & & & & & & 53,92 & 4,63 & & & 53,40 & 4,18 & & & 47,00 & 4,42 & 51,25 & 6,30 \\
\hline 15 & & & & & & & 58,55 & 3,63 & & & 64,33 & 7,85 & & & 52,86 & 6,22 & 56,75 & 7,29 \\
\hline 16 & & & & & & & 65,11 & 4,61 & & & 75,50 & 4,50 & & & 55,80 & 5,49 & 58,33 & 6,85 \\
\hline 17 & & & & & & & 71,00 & 4,80 & & & 76,00 & 0,00 & & & 60,80 & 6,27 & 56,50 & 0,50 \\
\hline 18 & & & & & & & 76,50 & 3,73 & & & & & & & 60,00 & 4,00 & 62,00 & 0,00 \\
\hline 19 & & & & & & & 82,67 & 2,05 & & & & & & & 64,50 & 4,50 & & \\
\hline 20 & & & & & & & & & & & & & & & 68,50 & 5,50 & & \\
\hline
\end{tabular}


Tabela II. Resultados do teste de Tukey comparando os diferentes tratamentos (temperatura e salinidade) empregados para avaliar a taxa de crescimento de carapaça $(\mathrm{mm})$ de Metamysidopsis elongata atlantica cultivado em laboratório (0 a 14 dias). Os tratamentos são: 1 $\left(13^{\circ} \mathrm{C}\right.$ e $\left.25 \%\right) ; 2\left(13^{\circ} \mathrm{C}\right.$ e $\left.30 \%\right) ; 3\left(13^{\circ} \mathrm{C}\right.$ e $35 \%$ ) $; 4\left(20^{\circ} \mathrm{C}\right.$ e $25 \%$ o) $5\left(20^{\circ} \mathrm{C}\right.$ e $30 \%$ o); $6\left(20^{\circ} \mathrm{C}\right.$ e $35 \%$ ); $7\left(25^{\circ} \mathrm{C}\right.$ e $25 \%$ ); $8\left(25^{\circ} \mathrm{C}\right.$ e $30 \%$ ) e $9\left(25^{\circ} \mathrm{C}\right.$ e $\left.35 \%\right)$. Os valores em negrito mostram diferenças estatísticas $(\mathrm{p}<0,05)$.

\begin{tabular}{cccccccccc}
\hline tratamentos & 1 & 2 & 3 & 4 & 5 & 6 & 7 & 8 \\
\hline 1 & & 0,955302 & $\mathbf{0 , 0 0 6 0 7 4}$ & $\mathbf{0 , 0 0 0 1 3 6}$ & $\mathbf{0 , 0 0 0 1 3 6}$ & $\mathbf{0 , 0 0 0 1 3 6}$ & $\mathbf{0 , 0 0 0 1 3 6}$ & $\mathbf{0 , 0 0 0 1 3 6}$ & $\mathbf{0 , 0 0 0 1 3 6}$ \\
2 & 0,9553017 & & 0,491967 & $\mathbf{0 , 0 0 0 1 3 6}$ & $\mathbf{0 , 0 0 0 1 3 6}$ & $\mathbf{0 , 0 0 0 1 3 6}$ & $\mathbf{0 , 0 0 0 1 3 6}$ & $\mathbf{0 , 0 0 0 1 3 6}$ & $\mathbf{0 , 0 0 0 1 3 6}$ \\
3 & $\mathbf{0 , 0 0 6 0 7 4}$ & 0,491967 & & $\mathbf{0 , 0 0 0 1 3 6}$ & $\mathbf{0 , 0 0 0 1 3 6}$ & $\mathbf{0 , 0 0 0 1 3 6}$ & $\mathbf{0 , 0 0 0 1 3 6}$ & $\mathbf{0 , 0 0 0 1 3 6}$ & $\mathbf{0 , 0 0 0 1 3 6}$ \\
4 & $\mathbf{0 , 0 0 0 1 3 6}$ & $\mathbf{0 , 0 0 0 1 3 6}$ & $\mathbf{0 , 0 0 0 1 3 6}$ & & 0,848101 & 0,998365 & $\mathbf{0 , 0 0 0 1 4 5}$ & $\mathbf{0 , 0 0 0 1 3 6}$ & $\mathbf{0 , 0 0 0 1 3 6}$ \\
5 & $\mathbf{0 , 0 0 0 1 3 6}$ & $\mathbf{0 , 0 0 0 1 3 6}$ & $\mathbf{0 , 0 0 0 1 3 6}$ & 0,848101 & & 0,997743 & $\mathbf{0 , 0 0 1 3 8 2}$ & $\mathbf{0 , 0 0 0 1 5 3}$ & $\mathbf{0 , 0 0 0 1 3 6}$ \\
6 & $\mathbf{0 , 0 0 0 1 3 6}$ & $\mathbf{0 , 0 0 0 1 3 6}$ & $\mathbf{0 , 0 0 0 1 3 6}$ & 0,998365 & 0,997743 & & $\mathbf{0 , 0 0 0 3 0 7}$ & $\mathbf{0 , 0 0 0 1 3 8}$ & $\mathbf{0 , 0 0 0 1 3 6}$ \\
7 & $\mathbf{0 , 0 0 0 1 3 6}$ & $\mathbf{0 , 0 0 0 1 3 6}$ & $\mathbf{0 , 0 0 0 1 3 6}$ & $\mathbf{0 , 0 0 0 1 4 5}$ & $\mathbf{0 , 0 0 1 3 8 2}$ & $\mathbf{0 , 0 0 0 3 0 7}$ & 0,901367 \\
8 & $\mathbf{0 , 0 0 0 1 3 6}$ & $\mathbf{0 , 0 0 0 1 3 6}$ & $\mathbf{0 , 0 0 0 1 3 6}$ & $\mathbf{0 , 0 0 0 1 3 6}$ & $\mathbf{0 , 0 0 0 1 5 3}$ & $\mathbf{0 , 0 0 0 1 3 8}$ & 0,992467 & 0,088457 \\
9 & $\mathbf{0 , 0 0 0 1 3 6}$ & $\mathbf{0 , 0 0 0 1 3 6}$ & $\mathbf{0 , 0 0 0 1 3 6}$ & $\mathbf{0 , 0 0 0 1 3 6}$ & $\mathbf{0 , 0 0 0 1 3 6}$ & $\mathbf{0 , 0 0 0 1 3 6}$ & $\mathbf{0 , 0 8 8 4 5 7}$ & 0,470855 & 0,470855 \\
\hline
\end{tabular}

entre cada muda), observando-se 20 mudas em salinidade de $30 \%$ e 18 em $35 \%$. Os resultados obtidos nos experimentos a $20 \pm 1{ }^{\circ} \mathrm{C}$ mostraram que as mudas ocorreram com períodos regulares (2,7 dias) e com pouca variação entre as salinidades (Tab. I).

A taxa de crescimento médio estimado de carapaça para misidáceos com idades entre 0 a 14 dias (idade de primeira maturação) foi $0,02 \mathrm{~mm} /$ dia, $0,04 \mathrm{~mm} /$ dia e 0,05 $\mathrm{mm} /$ dia para animais cultivados a $13^{\circ} \mathrm{C}, 20^{\circ} \mathrm{C}$, e $25^{\circ} \mathrm{C}$, respectivamente (Fig. 1).

A maior longevidade ( 86 dias) foi observada para misidáceos cultivados a $13 \pm 1^{\circ} \mathrm{C}$ e $25 \%$, enquanto que a $25 \pm 1{ }^{\circ} \mathrm{C}$ e $25 \%$ apresentaram menor sobrevivência (40 dias) (Tab. I).

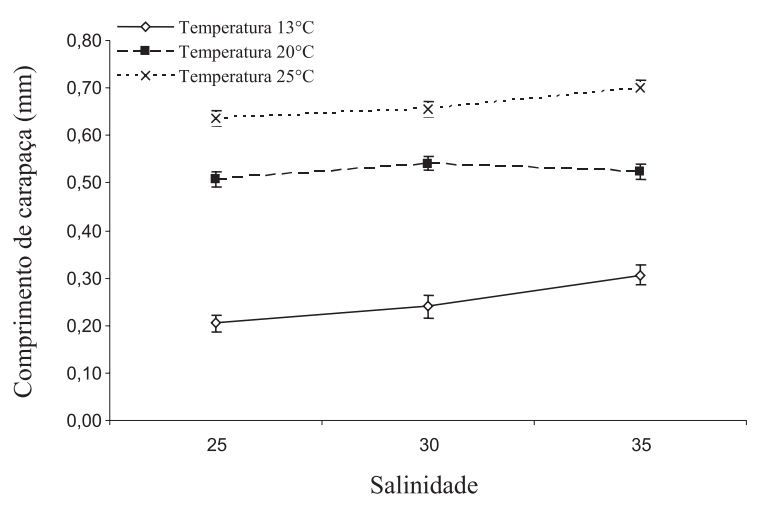

Figura 1. Taxa de crescimento de carapaça (mm) e \pm desvio padrão de Metamysidopsis elongata atlantica cultivado em laboratório ( 0 a 14 dias) em temperatura de $13,20,25 \pm 1^{\circ} \mathrm{C}$ e salinidade de $25,30,35 \%$

\section{DISCUSSÃO}

Clutter \& Theilacker (1971) descreveram que um único indivíduo de Metamysidopsis elongata (Holmes, 1900 ), cultivado em temperatura entre 14 e $20^{\circ} \mathrm{C}$, realizou 21 mudas em 157 dias. No presente trabalho registrou-se que, para organismos individualizados de M. elongata atlantica mantidos à temperatura $20 \pm 1^{\circ} \mathrm{C}$, o número total de mudas variou entre 19, 12 e 17 para diferentes salinidades de 25, 30 e 35\% (Tab. I). Estes resultados foram similares aos de ClutTER \& TheILACKer (1971), concordando com GAMA et al. (2001) que citam 12 mudas para 60 dias de cultivo de M. elongata atlantica a 30\%.

Os exemplares cultivados a $13^{\circ} \mathrm{C}$ não apresentaram desenvolvimento do marsúpio até o final do experimento, em todas as salinidades testadas. Este fato foi observado por McKenney (1996), que constatou que o tempo de aparecimento do marsúpio e a liberação da primeira ninhada estão diretamente relacionados com a temperatura. Este autor afirmou ainda que com o aumento da temperatura, a idade da primeira reprodução decresce, podendo ainda ocorrer a produção de mais juvenis na ninhada. Reitsema \& NefF (1980) reportam que Mysidopsis almyra (Bowman, 1964) maturou sexualmente entre 17 e 21 dias a $28^{\circ} \mathrm{C}$, e McKenney (1996) registrou que, para a mesma espécie, maturar em temperatura de $19^{\circ} \mathrm{C}$ foram necessários 20 a 25 dias. Domingues et al. (1999) relataram, para Mysidopsis almyra cultivado em laboratório a 26,22 e $18^{\circ} \mathrm{C}$, que o tempo da primeira desova - 13, 21 e 28 dias respectivamente - foi modificado em função da temperatura, aumentando com o decréscimo da mesma. No presente trabalho foram registradas cinco mudas até a maturação sexual (14 dias) em todas as salinidades e com temperatura de $20^{\circ} \mathrm{C}$, concordando com estudos realizados por GAMA et al. (2001) para M. elongata atlantica. Loureiro-Fernandes \& GAma (1996) registraram resultados semelhantes para Metamysidopsis munda (Zimmer, 1918), ocorrente na mesma região de estudo: verificaram cinco mudas até a maturação sexual, com um intervalo médio entre elas de 2,2 e 2,7 dias (machos e fêmeas), a uma salinidade de $30 \%$ e temperatura de $20^{\circ} \mathrm{C}$.

O crescimento médio diário em carapaça variou entre as temperaturas e salinidades estudadas. A taxa de crescimento da carapaça calculada no período de 0 a 14 dias (maturidade sexual) foi de $0,02 \mathrm{~mm} / \mathrm{dia}, 0,04 \mathrm{~mm} / \mathrm{dia}$ e $0,05 \mathrm{~mm} /$ dia a 13,20 e $25^{\circ} \mathrm{C}$, respectivamente. Indivíduos de $M$. elongata atlantica cresceram $0,04 \mathrm{~mm} /$ dia no período de 0 a 14 dias em $20^{\circ} \mathrm{C}$ e salinidade de $30 \%$, valores semelhantes aos encontrados por GAMA et al. (2001); a taxa máxima de crescimento encontrada para a espécie no presente trabalho foi de $0,0131 \mathrm{~mm} / \mathrm{dia}$ ( $58 \mathrm{dias}$ ). Os valores obtidos são sete vezes menores do que os descritos por ASTTHORSSON \& RALPH (1984) para Neomysis integer, a $16^{\circ} \mathrm{C}$ e salinidade de $30 \%$ no período de 60 
dias. Domingues et al. (1999) reportaram que, em experimentos testando diferentes temperaturas realizadas com Mysidopsis almyra, apresentaram taxas de crescimento significantemente maiores em $26^{\circ} \mathrm{C}$, quando comparadas com temperaturas de 18 e $22^{\circ} \mathrm{C}$.

Neste trabalho, os exemplares cultivados a $13 \pm$ $1^{\circ} \mathrm{C}$ e salinidade $25 \%$ obtiveram maior longevidade (86 dias) entre todos os tratamentos, enquanto os criados a $25 \pm 1^{\circ} \mathrm{C}$ e $25 \%$ apresentaram menor sobrevivência (40 dias). Isto pode ser explicado pelo fato dos organismos baixarem seu metabolismo quando submetidos a baixas temperaturas, reduzindo, desta maneira, o processo de envelhecimento biológico. REITSEMA \& NEFF (1980) defendem que a temperatura é um fator crucial no crescimento, maturação e reprodução de Mysidopsis almyra, um misidáceo que habita águas estuarinas da Flórida e costa oeste do México, locais onde a temperatura é mais elevada; os mesmos autores observaram ainda que $M$. almyra praticamente cessou sua reprodução quando a temperatura caiu de 25 para $20^{\circ} \mathrm{C}$, tendo sua reprodução aumentada com a elevação dos valores de temperatura para $30^{\circ} \mathrm{C}$. Os resultados das combinações de temperatura e salinidade aqui mostradas corroboram com os dados preliminares obtidos por GAMA et al. (2001) para $M$. elongata atlantica, destacando a ação da temperatura sobre o período de intermudas e crescimento da carapaça em misidáceos, independente das salinidades testadas.

Agradecimentos. À Fundação Universidade Federal do Rio Grande pelo apoio na realização das coletas; aos técnicos e docentes do Laboratório de Crustáceos Decápodes e ao M.Sc. Luiz Felipe Cestari Dumont, pelo auxílio e sugestões.

\section{REFERÊNCIAS BIBLIOGRÁFICAS}

Astthorsson, O. S. \& Ralph, R. 1984. Growth and moulting of
Neomysis integer (Crustacea: Mysidacea). Marine Biology 79:55-61.

Clutter, R. I. \& Theilacker, G. H. 1971. Ecological efficiency of a pelagic mysis shrimp: estimates of growth and mortality studies. Fish Bulletin 69:93-115.

Domingues, P. M.; Turk, P. E.; Andrade, J. P. \& Lee, P. G. 1999. Culture of the mysid, Mysidopsis almyra (Bowman) (Crustacea: Mysidacea) in a static water system: effects of density and temperature on production, survival an growth. Aquaculture Research 30:135-143.

Gama, A. M. S. \& ZAmboni, A. J. 1999. Aspectos da biologia e do cultivo de Metamysidopsis elongata atlantica para uso em testes de toxicidade. Nauplius 7:127-139.

Gama, A. M. S.; Montú, M. A. \& Gusmão, L. F. M. 2001. Ciclo de vida pós-marsupial e crescimento de Metamysidopsis elongata atlantica (Crustacea, Mysidacea, Mysidae) em cultivo de laboratório. Iheringia, Série Zoologia, 92(1):77-83.

Loureiro-Fernandes, L. \& Gama, A. M. S. 1996. Molting cycle in Metamysidopsis munda (Crustacea: Mysidacea). Nauplius 4:171-172.

Lussier, S. M.; Kuhn, A.; Chammas, M. J. \& Sewell, J. 1988. Techniques for the laboratory culture of Mysidopsis species. Environmental Toxicology Chemistry 7:969-977.

Mauchline, J. 1976. The Hiatt growth for diagram for Crustacea. Marine Biology 35:79-84.

1977. Growth of shrimps, crabs and euphausiids. Journal du Conseil. Consei Permanente Internacional pour L'Exploration de la Mer 37:162-169.

McKenney, C. L. 1996. The combined effects of salinity and temperature on various aspects of reproductive biology of the estuarine mysid, Mysidopsis bahia. Invertebrate Reproduction and Development 29:9-18.

NAIR, K. B. 1939. The reproduction, oogenesis and development of Mesopodopsis orientalis Tatt. Journal of Experimental Marine Biology and Ecology 9(4):175-223.

Nimmo, D. R. \& Hamaker, T. L. 1982. Mysids in toxicity testing, a review. Hydrobiologia 93:171-178.

Reitsema, L. A. \& NefF, J. M. 1980. A recirculating artificial seawater system for the laboratory culture of Mysidopsis almyra (Crustacea, Peracarida). Estuaries 3:321-323.

Stat Soft, Inc. 2001. STATISTICA, version 6.0. Tulsa, Stat Soft Inc.

Recebido em outubro de 2003. Re-submetido em outubro de 2005. Aceito em novembro de 2005. ISSN 0073-4721

Artigo disponível em: www.scielo.br/isz 